large extent their antigenic efficiency. Further, there is no necessity to kill them by heat. They die rapidly when suspended in 0.5 per cent. phenol saline. The less heat used for killing antigens the better. Staphylococci require thirty minutes at $58^{\circ} \mathrm{C}$. Streptococci, pneumococci, and Micrococcus catarrhalis require fifteen to twenty minutes at this temperatare. My antigens are all counted on I'homa slides. I obtain the fresh human blood from the patients by running it through a suitable needle from a vein in the forearm into bottles containing broken glass; it is defibrinated by shaking.

Turning now to the treatment of uncomplicated influenza, two types are definable: (1) The patient is able to carry on his work, but feels abnormally tired and off his food, lias. slight headache behind his eyes and at the back of his head and some backache and soreness of the limbs, and (2) the patient suddenly collapses with a high temperature, pink conjunctivae, ard all the usual symptoms.

If such cases are given at once $2 \frac{1}{2}$ million pure influenza vaccine, followed at a suitable interval by 5 million, the disease is very rapidly cut short, often in twenty-four hours. In other cases further doses ( $7 \frac{1}{3}$ and 10 million) may be necessary. Post-influenzal debility, which is so diffionlt to treat, will rapidly yield to a sinailar course of treatment. Quite recently I have had two very remarkable cases.

A boy of 18, whom I had cured of a post-pneumonic chronic infection of the lung, har a mysterious temperature of $99.6^{\circ}$ to $101^{\circ}$ for months. It had come on after a second dose (50 million) pure influenze antigen. I greatly feared it might be tubercufosis, but he failed to react to tuberculin (H.T.S.), and the catarrh vaccine previously used produced no result. 'Thinking it might be due to a chronic inftuenzal infection, I gave him 21 million pure influenza vaccine; the temperature began to fall slowly during some days, but at the end of a week it began to rise again. I repeated the dose, when the temperature fell suddenly, and the patient is making a rapid recovery.

suddenly, and the patient is making a rapid recovery. $100^{\circ}$ to A medical student had a temperature for some days of $100^{\circ}$ to $102^{\circ}$ F. before taking to his bed. Then he began to see double. He became delirious and had hallucinations, but when talked to would rouse up and talk fairly sensibly. The conjunctivae were congested; be was badly constipated and wetted his bed; he had, in short, all the symptoms of encephatitis lethargica. On the chance that it might be influeuzal I gave him 2.2 million iufluenza vaccine; the improvement was immediate. I followed it by 5 und $7 \frac{1}{2}$ million, after which his mind was normal, but the temperature was still $99^{\circ}$. I then gave him 10 million, and all the old delirium returned, especially that night, although he liad no further rise of temperature. He did not become mentally quite normal for two days. This reaction made it certain that the influenza bacillus or toxin was infecting his brain.

The blood of many cases who have suffered from influenza agglutinates the influenza bacillus sometimes in quite high dilution. The blood of one dactor who had had influenza some months before agglutinated the bacillus very strongly in a dilution of 1 to 160 . As a vule, however, the agglutination is only given in much lower dilution.

The evidence, then, that the influenza bacillus is the cause of the disease is that-

1. The microbe appeared with the appearance of the epidemic.

2. It was isolated, when the conditions were suitable, from a large number of cases of the disease.

3. A pure influenza bacillus antigen produced a marked degree of prophylaxis even in the small doses used.

4. It rapidly cuts short the disease when the infection is uncomplicated; and cures the after-effects.

5. A pure intluenza antigen can produce all the symptoms of the disease.

6. The serum of influenza patients agglatinates the influenza bacillus.

Complicating Infections.

I think in the recent epidemic there were two mothods of infection with the complicating catarrh microbes. Either they accompanied the influen $\%$ microbe when it was passed on from one person to the other or the patient's own microbes flared up when his resistance was lowered by the influenza bacillus. 'I'there was no uniformity in the complicating wicrobe Hora. Nearly all the worst cases of septic pneumonia had the Staphylococcus aureus, as well as streptococci, pneumococci, or Mirrococcus flirus, or other Gram-negative cocci. The staphylococci are much the most to be dreaded.

The incubation period of these microbes is longer than that of the influenza bacillus, and the human organism can often overcome them if the help of the influenza bacillus can be done away with in time; therefore, if the patient is given a $2 \frac{1}{2}$ million dose of influenza vaccine at once, complications are prevented. If there are marked signs of catarrh-indeed, if there is sputum at all-it is highly desirable to give a mixed vaccine containing Staphylococcus aureus, streptococci, pneumococci, and $M$. flavus, as well as the influenza vaccine. The initial dose should be :

$$
\begin{aligned}
& \begin{array}{lllll}
\text { Staphylococcus } & \ldots & \ldots & \ldots & 5 \text { million } \\
\text { Strepto-pneumococcus } & \ldots & \ldots & 17
\end{array} \\
& \begin{array}{lccccc}
\text { Strepto-pneumococcus } & \ldots & \ldots & 11 & \\
\text { M. caturrhalis } & \ldots & \ldots & \ldots & 1 z & " \\
\text { B. influerzae... } & \ldots & \ldots & \ldots & 23 &
\end{array}
\end{aligned}
$$

I always plate out the sputum or swab from the throat and get an autogenous mixed antigen as soon as possible. I also give the patient daily intravenous injections of diosal (1 per cent. sodium salt of di-iodosalicylic acid), beginning with $3 \mathrm{c.cm}$. in an average case. This substance is a very powerful intravital germicide for such microbes. The result of the combined method of treatment is often very remarkable and rapid.

Delayed resolution after the pneumonia is frequent. This can, as a rule, be rapidly cleared up with thiosinamine sodium salicylate (fibrolysin) or thiosinamine ethyl iodide (tiodine)

At the first sign of bronchial congestion, with cyanosis and dilatation of the right side of the leart, the patient must be given $\frac{1}{2}$ to $1 \mathrm{c.cm}$. doses of pituitrin as often as is necessary. This tones up the heart and bronchial muscles, or relaxes the latter if there is spasm. There is no other substance that gives this remarkable combined effect.

Reference

1 Lancet, November 23rd, 1918.

\section{THE TREATMENT OF INFLUENZA.} BY

WIILIAM D. D. SMALL, M.D., F.R.C.P.EDIN., Captain R.A.M.C.(T.C.) AND

W. O. BLANCHARD, M.D., Captatn M.C., U.S.ARMr.

During the second phase of the epidemic-in the two months from October 10th to December 9th, 1918-there were admitted to a general hospital, B.E.F., France, 937 cases of influeuza. The mortality wass 26 (2.77 per cent.). We have reasou to believe that this tigure compares very favourably with that obtained in other places, and it may therefore be of interest to outline the treatment omployed.

The first wave of the influenza epidenic terminated in the area towards the later part of the month of June. During July, August, and September only a few sporadic cases were seen. Early in October, however, the disease reappeared in a more virulent form, and in certain dis. tricts the mortality from it was very high. Nearly every case presented pulmonary symptoms in some degree, and at the commencenent of the wave were serious in about one ont of every four. In many there was a profound degree of toxaemia and a tendency to cardiac failure. The pulwonary complications included bronchitis, pleurisy bronchopneumonia, and lobar pneumonia, and oedema of the lungs. Other complications weve rare, but there were a few instances of nepluitis, and one of purulent mening. itis. Jelirium, unconsciousness, and extreme tremor were frequently present in the severer types. It will therefore be apparent that the series of cases with which we are dealing included a large proportion of the wost virulent infections. As practically all were admitted locally, they were admitted early - it great advantage, as each patient travels very badly.

The treatment in the acute stage may be related under síx headiugs :

1. The general management was that of a forer. As far as possible, patients should be treated in the fresh air. In this haspital they were placed in large Adrian huts, the doors and windows of which were always kept widely open. A sufficiency of blankets and lot bottles were provided to ensure warmth, but overheating (which produces restlessness) must be avoided. The diet should consist mainly of fluids, mills, boef-tea, clear soups, eggflips, jellies and custards. Beef-tea is especiafly valuable 
because of its stimulant properties. The drinking of large quantities of water, barley water, or lemon water, should be encouraged, as elimination is thereby promoted and the degree of toxaemia reduced.

2. Absolute rest during the acute stage is of paramount importance. In the severer cases collapse, shown by laboured breathing, cyanosis, exhaustion, sweating, and rapid acceleration of the pulse, is produced by any degree of movement. The patient should be disturbed as little as possible by examination, which should always be as brief as is consistent with efficiency; however desirable it may be from an academic point of view to determine the precise nature and extent of the pulmonary complications, their exact diagnosis matters little so fac as treatment is concerned, for this is indicated by the general condition of the patient, rather than by the pathological lesion present.

3. Sleep must be ensured. The result of a sleepless night at once shows itself by a falling off in the patient's general condition, and two or three such nights may make all the difference between recovery and death. Esuecially is sleep necessary in patients showing pronounced tremor; when this symptom is present the physician's first endeavour must be the production of sleep. He should regard tremor as an indication of exhaustion and a danger signal which he must not disregard. It has been our custom never to allow a sleepless night. Tepid sponging may first be tried, but, if insufficient, the patient should be given heroin gr. I's bypodermically, repeated once or twice, if necessary, at intervals of an hour and a half, until sleep is obtained. A satisfactory night's rest is almost always followed by considerable improvement in the general condition.

4. Medicinal Treatment.-We had had ample opportunity in the first stage of the influenza epidemic of testing the action of various medicinal agents, and were not satisfied that any of them exercised much influence on the course of the disease. At the commencement of the second wave we determined to test systematically certain likely remedies with the view of determining, if possible, their relative value, and of enabling us to adopt as routine treatment that which appeared to give the best results. Accordingly, groups of patients-fifteen in each-were put upon different drugs, and the progress of the various groups noted and compared. Aconite, aspirin, sodium salicylate, belladonna, arsenic quinine, Dover's powder, and gelsemium were tested in this way. The results were most striking. The patients treated by gelsemium im. proved in a manner far exceeding those given any other treatment. They stated that after a few doses their head. ache and backache had been much relieved, and that they felt greatly better in every way. "In most the temperature speedily commenced to fall, and the improvement in the general condition was obvious. So great was the contrast that it is no exaggeration to say that it was usually possible, without previous knowledge, to pick out the cases treated with this remedy. Of the other drugs tested, belladonna showed evidence of beneticial action in a number of cases, but none of the other diugs appeared to have the slightest influence. The patients were not selected in any way, but were taken consecutively as they were admitted; moreover, we were working separately at the time, and came to the same conclusions independently. The same observation was also made by the sisters in charge of the wards, who enthusiastically supported gelsemium against the other remedies.

We are well aware of the fallacies attending judgement of the action of remedies, and have therefore endeavoured to examine very critically our original observation. For this reason we have delayed drawing attention to the beueficial action which we believe gelsemium to exert. The test was repeated with the same result-again so striking that thereafter we did not feel justified in using any other remedy. Seeing, however, that belladonna appeared also to be of value, we considered it advisable to use it in combination. All our recent cases, therefore, have been treated with the following:

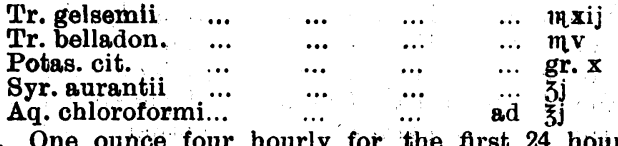

Sig. One ounce four hourly for the first 24 hours; ture is normal.
Potassium citrate was added as a mild diuretic. When the temperature reaches the normal the remedy should be stopped.

Gelsemium has previously been recommended as a remedy for influenza. ${ }^{1}$ Our observations lead us to believe that it exerts a marked beneficial action on the course of the disease, that it tends to shorten the illness, and it un. doubtedly relieves-and rapidly-the discomfort of the patient. Beyond slight ocular disturbances in a very small number of cases, we have not seen any disadvantages in its use.

5. Stimulants are indicated in the severe toxic cases; where the pulmonary symptoms are severe; where tremor is marked; where the pulse is unstable; and when the patient is obviously collapsed. Alcoliol in the form of brandy, whisky, or champagne gives the best results, and the large quantities that can be consumed without producing any signs of inebriation is very remarkable. The usual dose required is half an ounce overy four hours, or more often if the case be urgent. Should there be any suggestion of overdosage, aromatic spirits of ammonium ( $\frac{1}{2}$ to 1 drachm) may be substituted: Tincture of digitalis ( $m$ v every four hours) seemed to be useful when there were any signs of cardiac failure. Caffeine, strychnine, adrenalin, and pituitrin produce no beneficial action of any kind.

6. The treatment of pulmonary complications calls for no special discussion, as it is carried out on the usual lines. It may be mentioned, however, that the compound tincture of chloroform and morphine ( $m \times$ every four hours) is particularly efficient in soothing the of ten troublesome cough, and that inbalations of menthol and benzoin greatly relieve the tightness in the cliest so frequently present.

Treatment during Convalescence.-The degree of debility produced by influenza of the recent type is often extreme, and is particularly associsted with circulatory symptoms-palpitation, coldness, and blueness of the extremities, and a tendency to syncopo-as well as marked general weakness. During convalescence a period of com. parative rest is indicated, and a nourishing diet with extras-milk, eggs, port wine, and stout or porter. Iron and arsenic, cod-liver oil, or some bitter tonic should be prescribed according to the requirements of each individual case.

REFERENOE.

1 Ellingwood, Prescribir, 1913, p. 275. $+$

\section{A NOTE}

\section{ON THE EPIDEMIOLOGY OF INFLUENZA} AMONG WORKLRS

IN GAS WORKs, IN A CORDITE FACTORY, AND IN A - TIN MINE.

By Captain A. GREgOR, R.A.M.C.(T.), SPEOIALIBT SANITARY OFFICER, FALMOUTH,

[THE following interim report to the Medical Research Committee is published now in the hope that observations of a similar kind may be collected elsewhere. Bacteriological and chemical analyses of the events here recorded are now in progress, with a view to finding how far the observed facts may be related to the action of some inhaled substance, either upon infective agents or upon the protective mechanisms of the respiratory tract as such.]

For many years it has been an accepted fact among men working in gaseous fumes that they are practically immune from nasal catarrh and respiratory diseases in general. Further, in some parts of the country it is the custom to take children suffering from whooping :cough to the nearest gasworks and expose them to the fumes emanating from the oxide of iron purifiers during the process of cleansing, and the parents of these children firmly believe that by doing so the attack is much mitigated.

With this widespread belief in mind, investigation has been made during the influenza epidemic among various classes of the population, to see if there were any sub. stantial grounds for this popular belief, and the case incidence has been ascertained among the classes of persons shown in the tables. 